Voix et Images

voixetimages

\title{
La part des labels et des marchandises dans les Grandes Marées
}

\section{Yves Thomas}

Volume 15, numéro 1 (43), automne 1989

Jacques Poulin

URI : https://id.erudit.org/iderudit/200814ar

DOI : https://doi.org/10.7202/200814ar

Aller au sommaire du numéro

Éditeur(s)

Université du Québec à Montréal

ISSN

0318-9201 (imprimé)

1705-933X (numérique)

Découvrir la revue

Citer cet article

Thomas, Y. (1989). La part des labels et des marchandises dans les Grandes

Marées. Voix et Images, 15(1), 43-50. https://doi.org/10.7202/200814ar d'utilisation que vous pouvez consulter en ligne.

https://apropos.erudit.org/fr/usagers/politique-dutilisation/ 


\section{La part des labels et des marchandises dans les Grandes Marées}

\section{par Yves Thomas, université Trent}

Les références aux marques de produits de consommation dans les Grandes marées de Jacques Poulin ${ }^{1}$ concrétisent et permettent la mise en scène du triomphe de ce que Guy Debord a nommé le règne autocratique de l'économie marchande ${ }^{2}$ dont dépend précisément la société du spectacle. Bien entendu, cette domination du spectaculaire est avant tout un fait économique dont il est difficile de mesurer le cheminement dans le corps social. Mais ce qui frappe chez Poulin, c'est que par l'exercice d'un attrait signifié pour les objets industriels, il est parvenu à en désigner les effets chez ses personnages - légèreté, neutralité, tranquille insignifiance. Commentant un épisode des Grandes Marées, Gilles Marcotte a remarqué que [t]oute fiction, dans l'auvre de Jacques Poulin, débouche sur une telle pauvreté, une telle insignifiance. Ses personnages ne concluent pas [...] ils disparaissent, ils s'en vont, se dissolvent, avec la même «douceur» $[\ldots]^{3}$. Et c'est bien par cette légèreté, cette «douceur», que Poulin rend plus pressante encore la question de l'autorité spectaculaire des objets devenus marchandises. Là où on pourrait s'attendre à une résistance, on trouve chez les personnages principaux de ses romans, qu'il s'agisse de Jimmy, Amadou, Noël ou Jack Waterman, l'accueil, voire le besoin, du reflet de cette autorité. Ginette Michaud a déjà, dans une perspective différente ${ }^{4}$, analysé tout le soin que Poulin met à décrire les objects, à accumuler, pour ainsi dire, les signes de leur insertion en société. Le principe qui ordonne, par exemple, la description minutieuse des recettes de pâte et de garniture à tarte que propose la boîte de biscuits Graham dans les Grandes Marées est celui d'exactitude5. De fait, entre

1 Les références proviennent des éditions suivantes: les Grandes Marées [GM], Montréal, Leméac, 1986 (Poche Québec); Volkswagen Blues [VB], Montréal, Québec/Ámérique, 1984 (Littérature d'Amérique); le Cour de la baleine bleue [CBB], Montréal, Éditions du Jour, 1979 (le Petit Jour).

2 Guy Debord, Commentaires sur la société du spectacle, Paris, Éditions Gérard Lebovici, 1988, p. 12.

3 Gilles Marcotte, «Histoires de zouaves», Études françaises, vol. XXI, no 3, hiver 1985-1986, p. 14. Reproduit dans Littérature et circonstances. Essais, Montréal, l'Hexagone, 1989.

4 Ginette Michaud, «Récits postmodernes», Études françaises, vol. XXI, n 3, hiver 1985-1986, p. 67-88.

$5 \mathrm{Au}$ point où une certaine critique y a vu un gadget. Dans son compte rendu des Grandes Marées, Réginald Martel (la Presse, 13 mai 1978, p. D-3) note au sujet du lien entre le texte de Poulin et la bande dessinée: [il] y a risque cependant que la simplicité devienne un procédé, une sorte de gadget. Assurément, mais le 
la tasse et un quart de chapelure Graham qui amorce l'énumération des étapes de la recette de la pâte et le quart de tasse de sucre granulé qui marque la fin de la recette de meringue, Poulin travaille de façon complexe: en partant toujours d'un objet familier, en dressant une liste détaillée de traits qui le rendent parfaitement reconnaissable, il cultive la banalité à l'excès. Il convient d'insister néanmoins sur le fait que Poulin rend l'objet banal par le moyen d'une présentation littéraire. Ainsi, au gré des énumérations et des références, le banal apparait tour à tour comme un moment familier et un moment de surprise. Mais ce n'est pas seulement le contraste entre le littéraire et le commercial qui frappera, c'est encore plus leur mélange. Il ne faut pas oublier, à ce propos, de noter que si le terme «banal» peut manquer de spécificité, il a au moins l'avantage de désigner ici ce qui relie l'acte d'écrire aux conditions sociales d'émergence de la marchandise.

Pour informer le quotidien, pour interroger le monde de la consommation et la publicité qui l'exalte, Poulin laisse une grande place au pouvoir de séduction des marchandises dont les étiquettes contribuent à dérober au regard tout soupçon d'origine et de travail en cours. L'enveloppe publicitaire, par son aspect spectaculaire, son irradiation, apparaît alors déchargée de tout souvenir de vie, de toute trace des relations sociales de travail comprises dans la marchandise. Les personnages de Poulin sont donc rarement plus que des spectateurs. Analysant la dimension spectaculaire de l'économie marchande, Debord écrit:

La conscience spectatrice, prisonnière d' un univers aplati, borné par l'écran du spectacle, derrière lequel sa propre vie a été déportée, ne connaît plus que les interlocuteurs fictifs qui l'entretiennent unilatéralement de leur marchandise et de la politique de leur marchandise. ${ }^{6}$

Dans ces conditions, on doit à Poulin de s'être soucié de préciser les signes de la promotion des objets réduits à leur aspect marchand. C'est l'idée, l'image, le dehors, l'icône commercial devant lesquels le spectateur ébloui se laisse aller. Ainsi défilent dans les Grandes Marées: une raquette de tennis Wilson, du chocolat en poudre Nestlé Quik (Poulin écrit Quick), une boîte de nourriture pour chats Puss'n Boots, un dictionnaire Harrap's, un pot de café soluble Nescafé, une carabine Remington-Whittmore, un flacon de gin de Kuyper et même un robot lance-balles de tennis de marque Prince. Objets multiples: comestibles, articles de sport, livres de référence, ils illustrent la part équivoque du paradis ${ }^{7}$ de Teddy Bear. Ouverte aux vents du commerce, l'île Madame est en fait proche de l'île de Robinson ${ }^{8}$. Selon Philippe Hamon, qui s'est interrogé sur le phénomène des expositions dans la littérature et l'architecture au XIX ${ }^{e}$ siècle, 1'île de Robinson,

procédé est aussi un indice de l'importance que prend la marchandise dans l'œuvre de Poulin. Ne pourrait-on pas parler plutôt de «précision»?

6 Guy Debord, op. cit., p. 168

7 Gilles Marcotte écrit: Teddy Bear sait et a toujours su qu'il n'y a pas d'autre lieu pour lui que ce Paradis terrestre équivoque [...]. Loc. cit., p. 15.

8 Lise Gauvin se réfere à Teddy Bear en termes d'un nouveau Robinson. «Une voix discrète», le Devoir, 29 avril 1978, p. 33. 
c'est un peu le champ de Mars de l'Exposition, les mêmes structures et fonctions s'y retrouvent: déambulation méthodiquement conduite, passage en revue d'objets artificiels ou naturels qui vont être sélectionnés en fonction de leur utilité, importance des regards et des spectacles, projet à la fois récréatif et pédagogique, organisation et étiquetage du magasin-univers, glorification du travail [...]. ${ }^{9}$

Hamon établit ici un rapport essentiel pour comprendre l'autorité des objets dans la robinsonnade. Il serait impossible en effet de saisir ce qui pousse Poulin à écrire des pages sur le rêve d'une communauté idéale si l'on perdait de vue que l'île Madame constitue tout de même la confirmation d'une accoutumance au spectacle des marchandises. Il n'est pas sans intérêt toutefois de souligner que les objets artificiels ou naturels sont sélectionnés dans les Grandes Marées bien plus en fonction de leur exposition imminente qu'en fonction de leur utilité. Il est clair ainsi que le retour au Paradis de Teddy Bear est grandement compromis. Issu d'une société de consommation, il ne parvient pas à y échapper.

Dans ces conditions, il convient de voir la retraite de ce Robinson des Grandes Marées comme un exil continu. Exil d'abord au milieu des marchandises et, ensuite, au milieu des autres exilés (Marie, Tête Heureuse, l'Auteur, l'Animateur) qui arrivent sur l'île Madame comme portés par les grandes marées de chaque mois. Il est entendu que, sur l'île, le traducteur travaille avec ferveur et émotion. Il n'est pourtant pas sur l'île tout à fait par choix. Il s'agit plutôt là d'un accident qui ne confirme pas par l'expérience ni par l'épreuve, mais par l'exclusion, la perfection d'un présupposé. Teddy Bear est exclu, il est exilé malgré lui, et Poulin laisse entendre que les sentiments rattachés à son travail révèlent une insuffisance. Le malaise se manifeste concrètement: réduit à développer la même idée, à en exploiter toutes les implications, à la tourner et à la retourner dans tous les sens, parfois jusqu'à l'épuisement, parfois jusqu'à la perturbation complète de sa vie quotidienne, Teddy Bear passe indifféremment entre les hommes et les choses.

Mais au commencement, Teddy Bear n'est-il pas seul sur l'île? N'y a-t-il pas chez lui au moins l'énoncé possible d'un projet d'utopie qui aurait pu le propulser au-delà de lui-même vers un idéal, quitte même à le laisser tomber comme une épave? Il n'en est rien pourtant. Poulin se concentre sur ce qu'on ne peut qu'entrevoir, au mieux, sur ce que la mémoire retient difficilement. Dans une déclaration déroutante de Jack Waterman dans Volkswagen Blues, on reconnaît cette montée de scepticisme, cette naissance de l'indifférence et de la torpeur désenchantée qui sont à la base d'une âpre prophétie: un jour, il ne restera plus que des commerçants sur terre. (VB, p. 32)

Si on peut rapidement présumer de l'éloignement de Teddy Bear de la société hi-tech, à regard plus rapproché on ne saurait être étonné qu'il en soit autrement. Sur l'île Madame, le personnage central des Grandes Marées 
n'échappe pas à la tentation de l'oubli et à la menace de l'amnésie collective, car il se laisse tout de même éblouir par les machines. On relève à cet effet ici et là dans le texte un certain goût pour les nouveautés électroniques dont le robot lance-balles est la meilleure illustration.

Il n'avait pas enlevé la bâche. Il s'empressa de le faire et Marie s'approcha [...] Le Prince lui fit immédiatement une forte impression. Il était noir mat et solidement bâti! (GM, p. 32)

Dans la réaction de Marie devant le robot, il y a la trace d'un oubli de soi devant la marchandise, l'approche de ce que révèle une porte sacrée, que l'on constate également chez Teddy Bear. Le voisinage d'un modernisme intemporel, d'un industrialisme et de la solitude quasi idyllique du traducteur travaillant au milieu de plantes met en avant une recherche sur les formes utiles et marchandes de la jouissance qui incite à reconnaître le triomphe de la valeur spectaculaire sur l'utilité. Il importe plus à Teddy de montrer à Marie le potentiel de son robot que de le mettre en action dans un échange avec lui. Contemporain et mécanique: tel est l'esprit de cet autrui mécanisé qui fait irruption de façon inattendue dans un décor conçu pour Robinson. Le premier réflexe face à ce rapprochement inexpliqué est de ne considérer que ce qui est immédiatement visible. Teddy veut faire admirer ce qu'il admire, il veut parler de la marchandise qui l'éblouit sans attendre de réponse et pour se maintenir dans la stricte superficie des choses. Debord explique:

Celui qui subit passivement son sort quotidiennement étranger est donc poussé vers une folie qui réagit illusoirement à ce sort, en recourant à des techniques magiques. La reconnaissance et la consommation des marchandises sont au centre de cette pseudoréponse à une communication sans réponse. 10

Debord décrit ici l'état d'aliénation qui pousse l'individu à chercher l'effacement de l'autre pour éviter le dialogue. Le tennis pourrait bien être l'occasion d'un tel dialogue. Pourtant, Teddy préfere se mesurer à une machine.

On met une cassette là-dedans, dit-il. On presse le bouton [...] et le Prince suit le programme qui est enregistré sur le ruban magnétique de la cassette [...]-C'est un adversaire redoutable, dit Teddy. Il ne commet jamais d' erreur. (GM, p. 33)

Cet adversaire est proprement spectaculaire. Sa performance conditionne à la contemplation, à la fascination et non à l'engagement dans une action. La mécanique du Prince éblouit Teddy, et c'est précisément ce sentiment qu'il cherche à communiquer à Marie pour qu'à son tour elle puisse en être éblouie. Une finition soignée, il était noir mat et solidement bâti, un système de propulsion de balles équipé d'un oscillateur, une capacité de cent vingt-cinq balles et une puissance réglée par un cerveau électronique, ces aspects de l'appareil produisent en effet une forte impression, si bien que l'agenouillement

10 Guy Debord, op. cit., p. 169. 
de Teddy devant le lance-balles au moment où il explique à Marie le fonctionnement du cerveau pourrait bien être considéré comme un geste de vénération. Et cette lecture particulière du geste ne serait pas tout à fait en dysharmonie avec la manière dont l'appareil est mis en scène aux yeux de Marie. Plus encore, il faut noter que le mode de présentation du lance-balles dans la trame narrative correspond à une formule de représentation publicitaire qui peut provoquer soit l'envie de l'achat, soit l'admiration en la faveur de son propriétaire. Celui qui possède cette marchandise rendue spectaculaire acquiert un pouvoir du fait même qu'il détient un moyen d'éblouir. Ainsi, la valeur d'échange du «Prince» domine sa valeur d'usage et sa capacité extraordinaire est contagieuse: J'aime beaucoup le tennis moi aussi, dit Marie, et je trouve que le Prince est très beau. Elle caressa furtivement la joue du traducteur. (GM, p. 32) Marie est visiblement touchée par les circonstances de cette intimité avec la puissance mécanique que lui propose Teddy. Est-il besoin de le rappeler? Le visuel domine; une vitrine s' interpose entre le sujet et l'objet.

En faisant sienne une notion de Debord, Gilles Lipovetsky a parlé d'une économie de la frivolité où la publicité sans frontières donne le ton à un hyperspectacle conditionné par une pratique du zapping et dont les principes sont le choc et la surprise. L'efficacité de ce spectacle qu'est la «pub», affirme Lipovetsky, tient à sa superficialité ludique ${ }^{11}$.

À ce propos, il est intéressant d'examiner un épisode du Cœur de la baleine bleue qui illustre bien la mise en place d'une manifestation similaire de séduction dans le texte. En descendant la côte de la Fabrique à Québec, Noël, le personnage principal, se laisse séduire par des vitrines de boutiques. Poulin lui fait dire d'une manière contemplative:
Il y avait une sorte de tendresse dans la lumière [...] un mouvement inverse s'était amorcé qui déroulait devant mes yeux une succession ininterrompue et bigarrée de vêtements multicolores, de dentelles fines, de porcelaines de Chine, de bijoux précieux, de sculptures esquimaudes, de parfums délicats, d'aquarelles, de lainages, de bibelots de toutes les sortes, tandis que le nom des boutiques résonnait obstinément dans ma tête [...]. (CBB, p. 37-38)

Cette phrase peut étonner chez un écrivain dont les phrases sont rarement longues et complexes. Mais la vision du personnage semble commander ici une approche différente. L'étalage commercial entraîne l'énumération. L'attention de Noël est appelée autant par la succession des marchandises que par les marchandises en tant que telles. Enveloppé dans la lumière, le personnage de Poulin voit défiler devant lui des produits de luxe qui, derrière une vitrine, interdisent toute tendresse. Si Noèl glisse à la surface des étalages, c'est son mouvement même qui impose ces marchandises dans leur superficialité. Car le regard mouvant est presque une condition sine qua non de la saisie des objets en tant que marchandises. Noël est devant ces vitrines de boutiques comme devant

11 Gilles Lipovetsky, l'Empire de l'éphémère, Paris, Gallimard, 1987, p. 223. 
un écran. Le zapping commercial lui procure le confort immédiat du renouvellement et du recommencement des images. C'est en fait ce qui rend les marchandises interchangeables, successives et spectaculaires.

Force est de reconnaître alors que la légèreté des dentelles a sur Noël autant d'effet que la fragilité des porcelaines. Ayant cédé à la tentation du mouvement, le regard ne s'arrête pas pour interroger les différences entre les marchandises de luxe. Au vrai, cette interchangeabilité toute visuelle des objets précieux nous permet d'y reconnaître une métaphore du prix. Pour caractériser davantage la nature de cette métaphore, il suffit de constater que le regard de Noêl est normalisateur et soutient dès lors l'opposition entre d'une part le travail, la production et d'autre part la consommation et le loisir. Oisif, Noël se promène en fait dans une sorte de galerie marchande où se répète la promotion enjouée d'une vie conçue comme un inventaire.

Arrivé devant la dernière boutique de la rue, il s'assied épuisé sur un banc public et, plongeant la tête dans ses mains, il éprouve l'impression mystérieuse de s'être vidé. Il est curieux que sa descente de la rue de la Fabrique puisse avoir pareil effet sur lui. Si cette impression peut être attribuable à la fatigue résultant de l'éblouissement des vitrines, il n'en reste pas moins que le personnage de Poulin n'est plus en mesure de distinguer ce qu'il voit de ce qu'il est. La perception se confond donc à la projection et met au jour un trésor de produits luxueux inappropriables, mais accessibles par la vue.

Un exemple tiré cette fois-ci des Grandes Marées peut également illustrer ce point. Il s'agit de l'histoire de l'écriture que raconte Marie et dont il est question dans le chapitre intitulé «Le coffre au trésor». Un homme sur une grève heurte un objet du pied. En creusant dans le sable, il découvre petit à petit qu'il s'agit d'un coffre. Plus le coffre se révèle, plus l'homme creuse avec ferveur. Ce faisant, il se met à imaginer des richesses de toutes espèces: [...] des pièces d'or, des bagues serties de diamants, des vieux poignards et des dagues, des diadèmes, des colliers de pierres précieuses, des vieilles cartes de pirates [...] (GM, p. 121). C'est sans doute par l'action des marées sur le rivage que le coffre se trouve ainsi enfoui dans le sable. L'homme de l'histoire de Marie a certes cet avantage de pouvoir rêver, mieux encore que Teddy, aux possibilités d'un trésor qui excite tout à la fois la convoitise et le goût pour le raffinement ${ }^{12}$. L'homme s'émerveille à la perspective de découvrir un entassement de pièces de monnaie et de pierres précieuses mêlées à des objets antiques. Et son empressement est en relation directe avec cette accumulation imaginée. Mais ce qui donne à cette énumération son intérêt, c'est qu'elle inscrit le merveilleux sous le mode commercial de l'étalage. Le trésor imaginé montre à quel point le scintillement de l'or et des pierres précieuses peut représenter une force motivante. Toutefois, de façon inattendue, l'enthousiasme fait place à la déception dès que le coffre, une fois dégagé et ouvert, révèle, à l'opposé d'un trésor, du linge

12 Jamais, pourtant, Teddy ne fait de tels rêves puisque la splendeur pour lui s'incarne plus dans le Prince. 
moisi et des vieux vêtements de femme. La première impression est ainsi corrigée, et le passage du luxe à la misère, de la chimère à la désillusion, de l'attraction à la répulsion indique, selon cette allégorie, le rôle révélateur de l'écriture. Le plus saisissant pourtant, par-delà la révélation, c'est la position de l'homme à l'instant de l'ouverture du coffre. À genoux par terre, il soulève anxieusement le couvercle; le cour battant, il regarde [...]. (GM, p. 122) Excité, désireux de voir, l'homme est trompé par les signes prometteurs d'une richesse. En vérité, le levain de cette écriture, c'est la rencontre du lecteur avec l'homme privé de parole, ébahi, révérencieux, attentif devant les promesses d'un trésor qui, de fait, ne lui réserve que des déchets.

Très exactement, Poulin apprend à voir un monde qui porte ses contradictions, ses poisons, ses séductions sans que l'on soit tout à fait en mesure d'en prendre pleine conscience à la simple perception des signes qu'il affiche. Il identifie donc certains objets qui ont pour lui valeur significative, mais cette panoplie, si l'on peut dire, malgré le potentiel d'évocations et de sensations qu'elle comporte pour les personnages, tient son pouvoir de communication de l'intensification des apparences. Tout se joue en fait à la surface des objets entre l'étrangeté de leur surgissement et la surprise de leur banalité.

À voir apparaître, par exemple, la marque de commerce Nescafé dans la scène où Teddy fait connaissance avec Marie, on peut rapidement conclure à une influence venue du pop'art. Mais à regard plus rapproché, on constate que le maniement de la marque de commerce joue, chez Poulin, un rôle bien différent dans la mesure où il n'y est nullement question d'une esthétisation de la consommation. En vérité, nous ne manquons pas d'indices qui nous permettent de comprendre comment l'évocation des labels dans les Grandes Marées est productrice de tensions.

Dans cette perspective, le fait que Poulin montre Teddy en train de ranger le pot de café soluble dans une armoire peut mieux s'expliquer. Avant même d'esquisser un geste qui pourrait le gêner devant Marie, Teddy dissimule le Nescafé pour préparer un café au percolateur. Une certitude au moins semble s'imposer dans cette scène: Teddy ne veut pas faire mauvaise impression sur Marie, ce qui revient à suggérer que cette dernière n'a pas eu le temps d'apercevoir le pot de café soluble. Telle quelle et avec l'éclairage particulier que lui donne Poulin, cette scène fait vivre l'approche d'un choix et la préférence accordée au produit le moins commercial. Mais en même temps, il convient de signaler que le lecteur a vu ce que Marie n'a propablement pas vu et de surcroît, il en a constaté la disparition à un point tel que l'on puisse dire que la dissimulation est totalement significative. Ainsi, la négation du Nescafé met l'objet luimême en valeur.

Il suffit de lire plus loin pour s'apercevoir que le Nescafé dans les Grandes Marées est plus qu'un café soluble destiné à être consommé. En effet, la différence signifiée dans le texte entre le café soluble et le mélange de café java et moka du percolateur tient à si peu de choses, un incident banal, que le défaut de consommation prend de l'importance. Aussi ne faut-il pas être étonné lorsque, une fois de plus devant le pot de Nescafé, Teddy trouve encore moyen 
de ne pas en boire. Au chapitre éloquemment intitulé «La tarte aux biscuits Graham», le héros de l'île Madame retourne dans l'armoire chercher le pot qu'il avait fait disparaître auparavant. L'idée lui vient pourtant de faire une tarte aux biscuits Graham dont on a déjà parlé et aussitôt le Nescafé est oublié. Résultat: le consommation est différée, voire niée par le consommateur même. Mais qu'en est-il alors du Nescafé puisqu'on a droit ici à une promotion par une banalisation, au surgissement d'un «plus» à partir d'une dénégation? Et comment comprendre cette scène? Car, en effet, le lecteur ne sait si le protagoniste habite le domaine banal de la vie de banlieue ou bien encore si le protagoniste sert de simple support à l'exposition d'une marque de commerce ou même, dans la mesure où Poulin insiste, si en Teddy Bear il n'est pas question d'interroger une résistance à la consommation. Comme réminiscence littéraire, on citerait volontiers un passage de l'Hiver de force de Réjean Ducharme dans lequel les personnages principaux, Nicole et André, commandent chez l'épicier: [...] du fromage tranché Kraft, du pain tranché Weston, [...] du lait hypnotisé JJJoubert, du sucre superfin St. Lawrence [...] et $d u$ café décaféiné Sanka ${ }^{13}$. Les produits évoqués sont semblables, mais la perspective de l'évocation est tout autre. Là où Poulin présente et souligne l'aspect marchand du produit par sa dissimulation, Ducharme mine sa commerciabilité en la raillant.

Devant l'acquis que représente une marque de commerce, Poulin montre que le signe est plus chose que la chose même. L'important, ici, est la mise en rapport des marchandises - les biens de consommation, de luxe ou pas, dans leur aspect marchand - avec la représentation littéraire.

La netteté de la référence à une marque commerciale provoque une sorte d'élévation du produit. Chaque référence est plus que la chose qu'elle parait être puisqu'elle en est également le signe. Et la familiarité de son apparition dans le texte adresse au lecteur un appel si étrange qu'il peut être compris à la fois comme l'expression limite de la pensée sur l'usage possible de la chose et comme inscription abstraite de la marchandise dans la vie sociale. À Poulin revient non le mérite de dénoncer l'illusion produite par la marchandise, mais plutôt celui d'en exploiter toute la force.

Il place son lecteur devant une évidence: l'exactitude de l'apparition de l'objet en tant que marchandise est de l'ordre du déjà vu. Parsemés ici et là à travers le texte des Grandes Marées, ces produits entraînent à la reconnaissance d'une familiarité qui, tout compte fait, dépayse.

13 Réjean Ducharme, l'Hiver de force, Paris, Gallimard, 1984, p. 114 (Folio). 